\title{
Optimization of DND Multi-Depot Split-Load Pickup-Delivery Problem
}

\author{
Abdeslem Boukhtouta ${ }^{1, a}$, Sujoy Ray ${ }^{2}$, Andrei Soeanu², Raman Pall ${ }^{1}$, Jean Berger ${ }^{3}$ \\ ${ }^{1}$ Defence Research and Development Canada, Centre for Operational Research and Analysis, Ottawa, Ontario, Canada. \\ ${ }^{2}$ International Safety Research Inc., 38 Colonnade Rd, Nepean, Ontario, Canada. K2E 7J6. \\ ${ }^{3}$ Defence Research and Development Canada-Valcartier, Quebec, QC, Canada.
}

\begin{abstract}
This paper presents a solution approach to optimize vehicle routes for a multi-depot, multi-vehicle, pickup and delivery problem over a large ground transportation network. More precisely, we address ground transportation of orders for the Canadian Department of National Defence using heterogeneous vehicle fleets. The fleets consist of limited number of organizational vehicles hosted at pre-established depots and commercial order delivery services. The proposed approach involves leveraging an insertion cost gradient-descent heuristic followed by a greedy randomized adaptive search procedure. Experimental results generated using the historical orders of the organization indicate that the developed approach is effective in handling a wide range of scenarios and may generate near-optimal vehicle routes with an annual transportation cost reduction between $7.7 \%$ and $16.7 \%$.
\end{abstract}

\section{Introduction}

The Canadian Department of National Defence (DND) is using a heterogeneous fleet of vehicles for the ground transportation of goods between various bases and depots requesting orders through a central system. An order is an amount (weight) requested for delivery from a location to another location, at a specific date with a given priority. The request processing system is depicted in Figure 1. The system is responsible to develop routes and allocate orders to deliver in time using DND's own vehicles and commercial services. The DND's fleet of vehicles is known as Green Fleet (GF), which includes 5-Ton Small trucks (ST) and larger Tractor-trailers (TT). These are in limited in numbers and only a subset of locations, namely depots, host the GF vehicles. The route of an employed GF vehicle must start and end at the same depot where it is hosted. Moreover, each GF route in a solution must be scheduled at weekly basis for the whole year.
Thus, a year-long schedule for a GF vehicle is cost effective if the vehicle carries consignments on a regular basis. As such, contracted commercial fleet vehicles, also known as CF, supplement the GF to offer timely delivery of orders to various locations not served by the GF.

The analysis of historical data over DND's transport network revealed a critical need to optimize the problem of route planning for GF vehicles and efficiently using CF services to minimize the annual order delivery cost. In this paper, the resulting optimization problem is treated as an extension of the classical Pickup and Delivery Problem (PDP) under overarching organizational constraints. Thus, we define the problem of Multi-Depot Pickup-Delivery Problem with Split Loads (MDPDPSL) as follows.

Given a set of nodes $(N)$ and a set of edges $(E)$ where $E$ is a relation of $(N \times N)$, the transport network is a complete graph $\mathrm{G}=(N, E)$. Each edge $\langle i, j\rangle \in E$ in the graph is a direct arc of distance $d_{i j}$ between nodes $i$ and $j$.

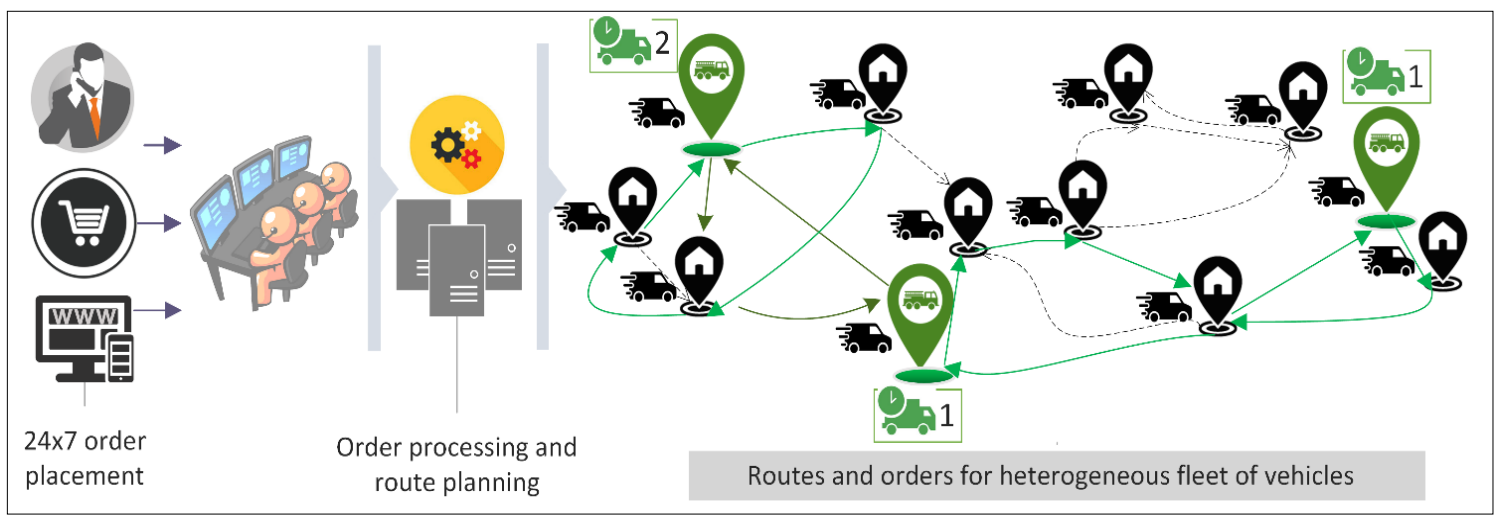

Figure 1: CAF's order processing and delivery system [2]

\footnotetext{
a Corresponding author: abdeslem.boukhtouta@forces.gc.ca
} 
All nodes can employ unlimited commercial vehicles with a defined service cost per $\mathrm{kg}$ per $\mathrm{km}(c f)$. A subset of these nodes is selected as depots $(D, D \subseteq N)$, where each depot can host a small number of GF vehicles. Each GF vehicle $k$ has defined carriage capacity $\left(Q_{k}\right)$ and service cost $\left(g f_{k}\right)$. The processing system receives continuous orders where each order $p$ is identified by a fixed amount $q_{i j}^{p}$, to be delivered from sender $i$ to receiver $j$. An order can also be split and delivered by multiple vehicles, if needed. The aim of the problem is to deliver all orders at minimum total routing cost. The main objectives of this paper are:

- Analyze exact requirements and constraints for order pickups and deliveries among DND locations;

- Devise an automated approach to generate nearoptimal solutions employing CF and GF fleets, using reasonable computing resources (time and memory);

- Evaluate the proposed approach using historical data from DND ground transportation network.

Manual order management over large transport network is often tedious, costly, error-prone and lacking process visibility. It also suffers from information and delays [1]. As such, end-to-end automation in order processing and delivery yields an improved supply chain quality control, efficient fleet use and notable cost reduction. The collected information allows better services and enhanced strategic decision making by applying state-of-the-art analyses. Also, seamless flow of such valuable information to-andfrom external partners can collaboratively improve the reliability and performance of the supply chain.

In the remainder of the paper, Section 2 presents the problem and details the benefits of collaborative use of Green Fleet and Commercial Services to improve order pickup and delivery. Section 3 highlights previous efforts on PDP and related research problems. Section 4 illustrates the proposed multi-phase approach. Thereafter, Section 5 discusses the results obtained over four scenarios using DND's order delivery historical data. Finally, Section 6 provides the concluding remarks.

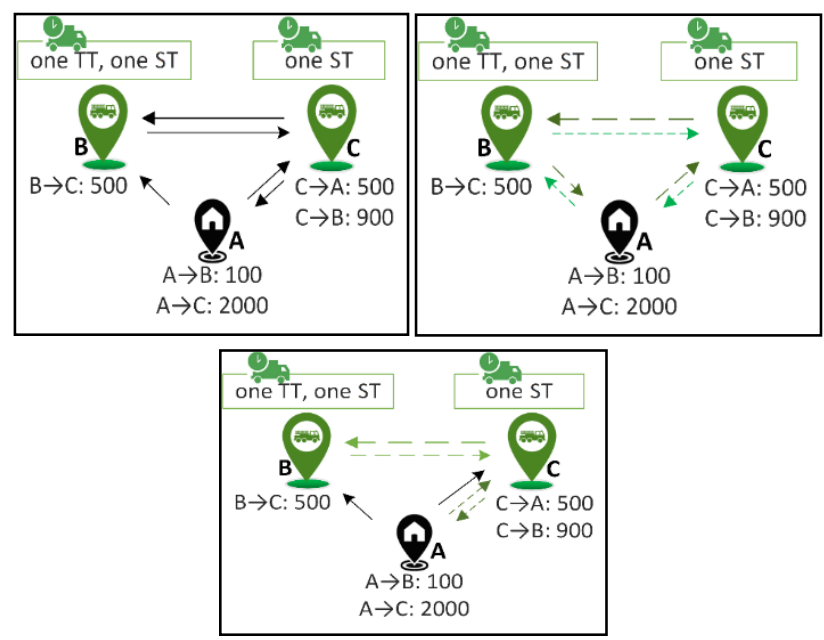

a)All CF Services (\$8) b) All GF services (\$4.5) c) Combined $\mathrm{CF} / \mathrm{GF}$ Services $(\$ 4.4)$

Figure 2: Comparison of solutions for the example [2]

\section{Problem Description}

Figure 2 shows the benefit of using different fleets from three locations A, B, C equidistant from each other by 100 $\mathrm{km}$. The orders among $\mathrm{A}, \mathrm{B}, \mathrm{C}$ are: $\left\langle\mathrm{A}, \mathrm{B}, 100, \mathrm{D}_{1}, 1\right\rangle,\langle\mathrm{A}, \mathrm{C}$, $\left.2000, \mathrm{D}_{1}, 1\right\rangle, \quad\left\langle\mathrm{B}, \mathrm{C}, 500, \mathrm{D}_{1}, 1\right\rangle, \quad\left\langle\mathrm{C}, \mathrm{A}, 500, \mathrm{D}_{1}, 1\right\rangle \quad$ and $\left\langle\mathrm{C}, \mathrm{B}, 900, \mathrm{D}_{1}, 1\right\rangle$. Each order has source, destination, weight $(\mathrm{kg}$.), order date and priority. We consider the same date and the same priority for all orders. B hosts $2 \mathrm{GF}$ vehicles: one ST (routing cost: $0.005 \$ / \mathrm{km})$ and one TT $(0.01 \$ / \mathrm{km})$ with capacity $900 \mathrm{~kg}$ and $4000 \mathrm{~kg}$ respectively. C also hosts an ST vehicle. We evaluate solutions that minimize total routing cost for a commercial cost of $0.0002 \$ / \mathrm{kg} / \mathrm{km}$.

Figure 2(a) depicts the solution with commercial deliveries of all orders. The total routing cost is $8 \$$. Figure 2(b) depicts a solution only using green vehicles efficiently where one TT is used from Node $\mathrm{B}$ on route: $\mathrm{B} \rightarrow \mathrm{A} \stackrel{2000}{\longrightarrow} \mathrm{C} \stackrel{900}{\longrightarrow} \mathrm{B}$ along with another $\mathrm{ST}$ vehicle from Node $\mathrm{C}$ on route: $\mathrm{C} \longrightarrow \mathrm{500} \rightarrow \mathrm{A} \rightarrow \mathrm{B} \rightarrow \mathrm{500} \rightarrow \mathrm{C}$. Each vehicle respects its carriage capacity and yields total routing cost $4.5 \$$. However, the solution cost can be further improved to $4.4 \$$ using green and commercial fleet together as shown in Figure 2(c). The proposed GF routes are: $\mathrm{B} \stackrel{500}{\longrightarrow} \mathrm{C} \stackrel{900}{\longrightarrow} \mathrm{B}$ and $\mathrm{C} \longrightarrow \mathrm{500} \rightarrow \mathrm{A00} \longrightarrow \mathrm{C}$. $\mathrm{CF}$ vehicles are used for additional shipment: $\mathrm{A} \stackrel{100}{\longrightarrow} \mathrm{B}$ and $\mathrm{A} \stackrel{1100}{\longrightarrow} \mathrm{C}$, marked by black arrows.

In practice, orders are grouped as per the schedules of the GF vehicles. Moreover, large orders are split in parts according to vehicles' availability and order priority. For example, two orders 〈A,B,100, 22/1/2018,1〉 and $\langle A, B, 2000,23 / 1 / 2018,2>$ are placed to the processing system on Monday and Tuesday. Priority 1 and 2 require them to be delivered within 1 and 2 week(s) respectively. If the vehicles are scheduled on Monday and Thursday between $\mathrm{A}$ and $\mathrm{B}$, the first order should be shipped on next Thursday while the second one can be shipped in next two consecutive schedules. Thus, on Thursday 25/1/2018, total combined transhipment between $\mathrm{A}$ and $\mathrm{B}$ will be $\mathrm{A} \stackrel{1100}{\longrightarrow} \mathrm{B}$.

Figure 3 depicts our five-step strategy for order grouping. First, sensitive cargo orders are removed since they cannot be delivered by commercial services. Then, each order is divided into multiple transhipments as per vehicle availability and order priority. Statistical methods are used to eliminate unexpectedly high or low orders (outliers) since they can be served using commercial services. It is not beneficial to have schedules accommodating spurious raise in orders. Finally, an expected weekly amount is determined for every source and destination pair using one standard deviation over the average order weight.

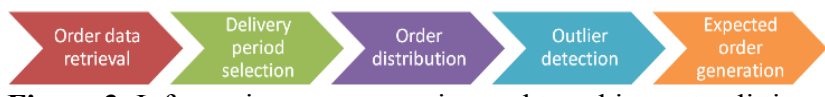

Figure 3: Information pre-processing and transhipment splitting

\section{Literature Review}

Pickup and Delivery Problem (PDP) and its variants are research problems with NP-Hard complexity. Nowak et al. [3] studied the impact of order splitting over single-depot 
PDP. Dividing an order over multiple vehicles needs less vehicles and saves transportation cost. However, according to our knowledge, impact of load splitting in multi-depot setting is very little assessed. Only RíosMercado et al. [4] discussed a specific PDP routing problem in multi-depot and split-delivery setting.

Vehicle Routing Problem (VRP) is a simplified PDP where pickup or delivery location is same for all orders [5]. In more general case, PDP has no restrictions on rearranging commodity in vehicles and no fixed location for pickups or deliveries [6,5]. PDP is studied in relation to VRP [7] for goods deliveries, mail service, passenger transport, etc. Dial-A-Ride Problem (DARP) is a form of PDP where customers call for a ride from one location to another. DARP uses constraints on vehicle capacity and delivery time-window [8]. In practice, additional constraints are also used. VRP with Backhauls (VRPB) places constraints such that all deliveries precede the pickups to avoid rearrangement of goods in vehicles. For patient transportation, VRP with LIFO mandates that every picked-up patient is dropped off to a hospital before another pickup [9]. A multi-trip PDP with time-windows and manpower planning is detailed in [10] where nonemergency ambulance transfer is discussed. Analytic techniques (e.g. branch-and-cut and branch-and-price) are also used for PDP and DARP [11]. In addition, heuristic algorithms for single and multi-depot VRP with pickup and deliveries (MDPDP) are employed in [7].

Tabu search is applied in [9] on VRPPD with LIFO. A hybrid metaheuristic using genetic algorithm is detailed in [12] for VRP with multiple pickups, single delivery and time windows (VRPMPDTW). A genetic algorithm for multi-vehicle and multi-depot pickup and delivery with time windows is detailed in [13]. In [14], the authors employed an approach, leveraging iterative modified simulated annealing with greediness, for VRPPD. Particle Swarm Optimization is applied in [15] for pickup and delivery with time-windows (PDPTW). MDPDP with split load (MDPDPSL) is addressed in [4] using a Greedy Randomized Adaptive Search Procedure (GRASP). However, greedy algorithms have limitations for large problems [16]. In our case, this challenge is addressed by using a quick heuristic first to find good order delivery routes from each location. Then, the routes are extended by inserting other nearby locations. Finally, the routes are efficiently mixed using a GRASP based metaheuristic.

\section{Proposed Approach}

Given the scale of DND ground distribution network and complex operational constraints, optimal solution search was challenging. Unlike classical VRP, in MDPDPSL, each node can be a point of order pickup and/or delivery for any other nodes. Analytical approaches, such as branch and bound, branch and cut, etc., were inadequate to handle instances of practical size and failed to provide solution in reasonable time due to large number of decision variables and nonlinear nature of the problem. Thus, we use Heuristic approach to intelligently construct feasible solutions that are iteratively enhanced and compared. To improve solution quality, geographic features of the locations are exploited. An initial route is often extended to include additional routing points close to its path. This allows exploiting more opportunities for pickup and delivery among visited nodes. GF routes, computed using expected order amount, must also be maintained for a whole year. Only appropriate orders can be assigned periodically to the scheduled vehicles of limited capacities.

\subsection{Problem Decomposition and Route Extension}

First, we decompose the core problem into instances of a Split-Delivery VRP (SDVRP) variant for each order pickup location and solve each instance near-optimally using heuristics. In SDVRP, orders are picked from one location and served to various locations as per delivery needs. However, unlike classical SDVRP, in our case, GF vehicle(s) can be called from some of $D(D \subseteq N) \operatorname{depot}(\mathrm{s})$ if the pickup location does not host adequate GF vehicles. Also, we allow split-delivery using heterogeneous fleets and make use of the availability of unlimited CF vehicles.

We extend an existing insertion cost gradient descent route construction heuristic. The technique initially computes an upper bound solution quickly as a reference using a nearest neighbour search. Then multi-tours are incrementally constructed by growing a pseudo-randomly selected tour with a nearby unserved node at a time. Two different supervision features guide the solution search: First, each multi-tour is stored in a sorted queue of fixed size in the ascending order of its total tour cost. Therein, a multi-tour of lowest cost is grown in each iteration. The fixed queue size also limits memory use and removes disqualified high cost multi-tours. Second, a map structure is maintained to record the serving costs for different fractions of total order serving. The map guides the solution search by offering early hint on whether a growing multi-tour has potential to produce a near-optimal solution. When a multi-tour serves all the nodes, it forms a solution. The lowest-cost solution is always retained and any multitour exceeding that cost gets eliminated from the sorted queue. The obtained solutions of all individual SDVRP instances are then combined to a final solution with vehicles starting from multiple depots.

In the route extension phase, each vehicle route is extended to add additional locations close to the route. The underlying method first finds nearest neighbours for each leg of a route within a cost threshold. Then, it adds a neighbour node into the route between two nodes if and only if the new routing cost through the inserted node does not exceed a fixed small percentage of the original routing cost between the two nodes. This allows efficient loading of GF vehicles with more orders between the nodes of the route where the pickup nodes are different from the original supply node of an individual vehicle path.

\subsection{GRASP and Vehicle Route Selection}

The GRASP step improves the combined solution by shifting order(s) from one vehicle to other when the latter passes through the same order pickup and delivery locations in sequence and has enough remaining cargo capacity. Efficient order loading helps reducing transport 
cost by eliminating redundant and inefficient $\mathrm{CF}$ and GF vehicles from the original solution. We iteratively perform pseudo-random order selection with a bias to its cost benefit ratio. GRASP depends on two strategies: Diversification and Intensification. First one involves a LocalSearch method to approximately evaluate cost benefits by transferring the selected order to available vehicles and determines the suitable one. Transfer of the order forms a new solution. Intensification is implemented through PathRelinking which improves both, the current best and the newly found solutions by exchanging their distinct features. Different order delivery strategies of previously found solutions are also analysed. This may lower the cost of both solutions. After each iteration, the current best solution is retained.

The cost of each route is further evaluated against the option of delivering associated orders using CF service. Then most efficient GF routes are selected from the depots, as per vehicle availability and vehicle reuse constraints. The remaining orders are then delivered commercially.

\subsection{Benefits and Drawbacks}

The proposed approach allowed handling large-scale problem instances of order deliveries in multiple phases. Decomposing MDPDPSL into SDVRP instances helped treating the main problem concurrently and with lower memory footprint per sub-problem. Due to unlimited CF vehicle availability, a valid solution is always guaranteed for any of our problem instances. Geographic positions of pickup and delivery locations also helped in cost saving by route extension. However, initial SDVRP routes are formed using recipients' locations for every sender. This reflects a bias to produce cost efficient routes from those locations sending large amount of orders but an initial subset of less efficient routes, computed from SDVRP instances, could be found highly efficient once more orders are assigned between nodes of the route. Moreover, lack of processing ability limits evaluation of route extension phase within a distance threshold from the initial route. Also, greedy approach (GRASP) to improve vehicle's delivery efficiency is fast but not always optimal.

\section{Results and analysis}

We tested the approach on four years historical order delivery data, across 308 DND locations including 14 depots. These locations are operational from Monday to Friday between 7:30am and 4pm. Unlimited CF and limited numbers of GF vehicles (ST and TT) are used in four scenarios. Each GF vehicle is scheduled to start from its depot on every Monday and/or Thursday of the week to maximize operation time of the locations. Vehicles returning to depot in two or three days are often reused for next weekly schedule. The travel costs of ST and TT are respectively $\$ 0.65$ and $\$ 1.00$ per $\mathrm{km}$ Commercial fleet charges $\$ 0.000229$ per $\mathrm{km}$ per $\mathrm{kg}$ in each scenario except the last one where an advanced cost estimation technique is used (will be described later). The time to unload and load orders at any base is considered as 1 hour. A full day off is also added for each driver's schedule after 5 days of work as per regulations. In this setting, the purpose of the scenarios are as follows:

- Scenario 1 minimizes total order delivery cost without any restriction on the selection of depots among all bases and availability of CF and two types of GF fleets.

- Scenario2 estimates the cost and route changes over Scenario 1 by closing Toronto (ON) base and shifting all its orders to Angus-Borden (ON) base in proximity.

- Scenario 3 aims to minimize the order delivery cost by specifying candidate depots and availability of green vehicles only from these depots: Angus-Borden (ON), Bagotville (QC), Cold Lake (AB), Comox (BC), Edmonton (AB), Esquimalt (BC), Gagetown (NB), Halifax (NS), Greenwood (NS), Montreal (QC) and Valcartier (QC). Among these locations, Comox hosts 1ST, Montreal hosts 4 TTs and 2 STs. Each of the other depots hosts only 1 TT. Furthermore, each ST route is restricted to a tour duration spanning no more than 2 days while adding $\$ 150$ for each overnight stay of ST truck. GF drivers are allowed up to 13 hours of driving within their daily schedule of 14 work hours.

- Scenario 4 assesses near optimal order delivery cost on the same constraints of Scenario 3 but alters the linear estimated rate of $\mathrm{CF}$ service with a more realistic market driven cost estimation method.

The estimation leverages a data under-sampling-technique that exploits k-nearest neighbours (k-NN) algorithm over historical data to predict the transport cost. First, Density Based Scanning (DBScan) finds a cluster of at least $k$ closest data points with their relative distances from the input order data in a multidimensional space. The scanning stops when $k$ neighbours are found in its largest cluster. The measured distance of these data points from input helps computing the order delivery cost. We notice that linear estimation is reasonably useful for orders weighing up to $2000 \mathrm{~kg}$., as the carriers compete to deliver the orders. However, for orders with weights over $2000 \mathrm{~kg}$., linear estimation is less accurate. The proposed method is useful for the entire range of weights, particularly for large weights. Figure 4 depicts total number of depots and GF vehicle tours (certain vehicles are reused) for every week in each of the scenarios. Table 1 details the experimental results. Four years of selected order deliveries actually costed DND \$34.5 million. The cost would increase to \$45 million if only CF services would be used at a linear cost of $\$ 0.000229$ per $\mathrm{km}$ per kg. However, using combined GF and CF services, significant saving can be achieved. Scenario 4 indicates a better CF service cost estimation technique (deviation: $\pm 5 \%$ of actual service cost) may save $13.41 \%$ to $16.75 \%$ of operation cost.

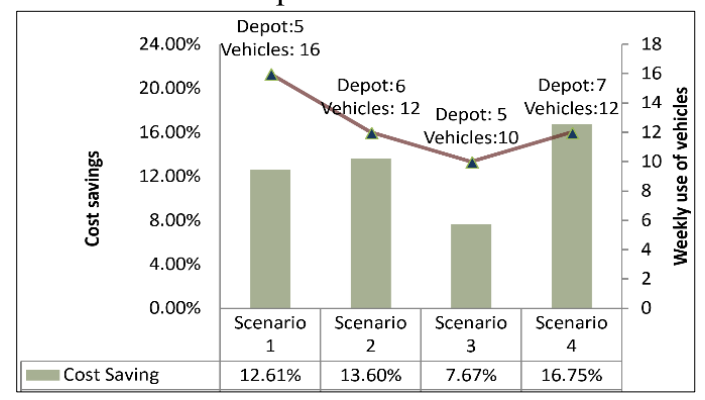

Figure 4: Cost saving and vehicle allocation details 
Table 1: Experiments on four years DND order delivery data

\begin{tabular}{|l|r|r|r|c|}
\hline \multicolumn{1}{|c|}{ Scenario 1 } & GF Cost (\$) & CF Cost (\$) & Total Cost (\$) & Savings (\%) \\
\hline Estimated all orders served commercially (Linear) & & & & \\
\hline Estimated current operational cost & 0 & $45,023,417$ & $\mathbf{4 5 , 0 2 3 , 4 1 7}$ & \\
\hline Actual current operational cost (database) & $7,546,240$ & $40,787,693$ & $\mathbf{4 8 , 3 3 3 , 9 3 3}$ & \\
\hline Estimated proposed operational cost: 1std (Linear) & $7,546,240$ & $26,968,443$ & $\mathbf{3 4 , 5 1 4 , 6 8 3}$ & \\
\hline Scenario 2 & $8,295,785$ & $21,866,053$ & $\mathbf{3 0 , 1 6 1 , 8 3 8}$ & $\mathbf{1 2 . 6 1}$ \\
\hline Estimated all orders served commercially (Linear) & & & & \\
\hline Estimated current operational cost & & $44,961,180$ & $\mathbf{4 4 , 9 6 1 , 1 8 0}$ & \\
\hline Actual current operational cost (database) & $7,635,680$ & $40,753,909$ & $\mathbf{4 8 , 3 8 9 , 5 8 9}$ & \\
\hline Estimated proposed operational cost:1std (Linear) & $7,546,240$ & $26,968,443$ & $\mathbf{3 4 , 5 1 4 , 6 8 3}$ & \\
\hline Scenario 3 & $6,010,020$ & $23,808,863$ & $\mathbf{2 9 , 8 1 8 , 8 8 3}$ & $\mathbf{1 3 . 6 0}$ \\
\hline Actual current operational cost with new constraints & & & & \\
\hline Estimated proposed operational cost (1std) & $7,546,240$ & $26,968,443$ & $\mathbf{3 4 , 5 1 4 , 6 8 3}$ & \\
\hline Scenario 4 & $7,925,069$ & $23,940,814$ & $\mathbf{3 1 , 8 6 5 , 8 8 3}$ & $\mathbf{7 . 6 7}$ \\
\hline Estimated all orders served commercially (Clustering) & & & & \\
\hline Estimated current operational cost (Clustering) & & $32,312,903$ & $\mathbf{3 2 , 3 1 2 , 9 0 3}$ & \\
\hline With commercial service overestimate correction (95\%) & & & $\mathbf{3 5 , 0 9 2 , 2 8 7}$ & \\
\hline With commercial service underestimate correction (105\%) & & & $\mathbf{3 3 , 7 1 4 , 9 8 5}$ & \\
\hline Estimated proposed operational cost (1std) - (Clustering) & $5,675,524$ & $23,057,140$ & $\mathbf{2 8 , 7 3 2 , 6 6 4}$ & $\mathbf{1 6 . 7 5}$ \\
\hline With commercial service overestimate correction (95\%) & & & $\mathbf{2 7 , 5 7 9 , 8 0 7}$ & $\mathbf{2 0 . 0 9}$ \\
\hline With commercial service underestimate correction (105\%) & & & $\mathbf{2 9 , 8 8 5 , 5 2 1}$ & $\mathbf{1 3 . 4 1}$ \\
\hline
\end{tabular}

\section{Conclusions and future work}

The proposed approach employs initial route generation and subsequent metaheuristic route improvement, yielding competitive solutions for relevant size PDP problems.

We examined four scenarios of growing difficulty in terms of depot locations, vehicle availability, routing constraints and cost estimation. The use of heterogeneous vehicles along with split loading provides notable cost savings due to improved capacity use in terms of cargo weight. Cargo volume is subject of future work though. The approach is suitable for transport management, aiming at cost effective order delivery by different types of vehicle with specific trade-offs in terms of routing cost versus capacity or loading level. As such, the approach is anticipated to allow DND to carry out mission planning with reduced distribution cost and improved sustainment.

\section{References}

1. Esker, Improving Customer Service and Fulfillment with Order Processing Automation, online, https:/quitpaper.esker.com/Gartner-ImprovingCustomer-Service-and-Fulfillment-documentlibrary.html (March 2019)

2. Boukhtouta A. et al., Modelling and Optimizing DND Ground Transportation Distribution Network, DRDC Scientific Report, 83 pages, (2019).

3. M. Nowak, A. Ergun, C. C. White, Pickup and Delivery with Split Loads, Transportation Science 42, 32-43, (2008).

4. R. Z. Ríos-Mercado, J. F. López-Pérez, A. CastrillónEscobar, A GRASP for a Multi-depot Multicommodity Pickup and Delivery Problem with Time Windows and Heterogeneous Fleet in the Bottled Beverage Industry, Springer,143-157, (2013).
5. M.W. P. Savelsbergh, M. Sol, The general pickup and delivery problem, Transp. Science 29, 17-29, (1995).

6. G. Berbeglia, J-F. Cordeau, I. Gribkovskaia, G. Laporte, Static pickup and delivery problems: A classification scheme and survey, TOP 15, 1-31, (2007).

7. G. Nagy, S. Salhi, Heuristic algorithms for single and multiple depot vehicle routing problems with pickups and deliveries, EJOR 162, 126-141, (2005).

8. J-F. Cordeau, G. Laporte, The dial-a-ride problem: models and algorithms, Annals of Operations Research 153, 29-46, (2007).

9. E. Benavent, M. Landete, E. Mota, G. Tirado, The multiple vehicle pickup and delivery problem with LIFO constraints, EJOR 243, 752 - 762, (2015).

10. A. Lim, Z. Zhang, H. Qin, Pickup and Delivery Service with Manpower Planning in Hong Kong Public Hospitals, Trans. Science 51, 688-705, (2016).

11. L. Xue, Z. Luo, A. Lim, Exact approaches for the pickup and delivery problem with loading cost, Omega 59, Part B, 131 - 145, (2016).

12. S. Yanik, B. Bozkaya, et al., A new VRPPD model and a hybrid heuristic solution approach for e-tailing, EJOR 236, 879-890, (2014).

13. E. B. Alaia, I. H. Dridi, H. Bouchriha, P. Borne, Optimization of the multi-depot \& Multi-vehicle pickup and delivery problem with time windows using genetic algorithm, IEEE-CoDIT, 343-348, (2013).

14. G. Martinovic, I. Aleksi, A. Baumgartner, Singlecommodity vehicle routing problem with pickup and delivery service, Mathematical Problems in Engineering (2008).

15. P. Sombuntham, V. Kachitvichyanukul, Multi-depot Vehicle Routing Problem with Pickup and Delivery Requests, American Institute of Physics Conf. Series, 1285, 71-85, (2010).

16. M. G. C. Resende, C. C. Ribeiro, Greedy Randomized Adaptive Search Procedures, Springer, 219-249, (2003). 